\title{
Chronic thromboembolic pulmonary hypertension: diagnosis, operability assessment and patient selection for pulmonary endarterectomy
}

\author{
Tom Verbelen ${ }^{1}$, Laurent Godinas ${ }^{2}$, Geert Maleux ${ }^{3}$, Johan Coolen ${ }^{3}$, Guido Claessen ${ }^{4}$, Catharina Belge ${ }^{2}$, \\ Bart Meyns ${ }^{1}$, Marion Delcroix ${ }^{2}$ \\ ${ }^{1}$ Department of Cardiac Surgery, University Hospitals Leuven, Leuven, Belgium; ${ }^{2}$ Department of Pneumology, University Hospitals Leuven, \\ Leuven, Belgium; ${ }^{3}$ Department of Radiology, University Hospitals Leuven, Leuven, Belgium; ${ }^{4}$ Department of Cardiology, University Hospitals \\ Leuven, Leuven, Belgium \\ Correspondence to: Tom Verbelen, MD, PhD. Department of Cardiac Surgery, University Hospitals Leuven, Herestraat 49, 3000 Leuven, Belgium. \\ Email: tom.verbelen@uzleuven.be.
}

\begin{abstract}
Healthcare providers outside pulmonary hypertension $(\mathrm{PH})$ centers having misinformation or insufficient education, and a general lack of treatment awareness contribute to a massive underdiagnosis of chronic thromboembolic pulmonary hypertension (CTEPH), diagnostic delay and refusal of surgery by patients. Together with the subjective operability assessment, this leads to too few patients undergoing pulmonary endarterectomy (PEA); even though this surgery results in improved survival and exercise capacity. Acute pulmonary embolism (PE) survivors should undergo a CTEPH screening strategy. Patients screened positive and those with CTEPH symptoms (with or without history of PE), should undergo transthoracic echocardiography (TTE) to determine the probability of PH. High PH probability patients should undergo a ventilation/perfusion (V/Q) scan. A negative scan rules out CTEPH. Patients with a positive V/Q scan, but also patients with findings suggestive for CTEPH on computed tomography pulmonary angiography (CTPA) to diagnose acute PE, should be referred to a CTEPH center. Further diagnostic work-up currently consists of catheter based pulmonary angiography, CTPA and right heart catheterization. However, new imaging technologies might replace them in the near future, with one single imaging tool to screen, diagnose and assess operability as the ultimate goal. Operability assessment should be performed by a multidisciplinary CTEPH team. PEA surgery should be organized in a single center per country or for each forty to fifty million inhabitants in order to offer the highest level of expertise. Informing patients about PEA should preferably be done by the treating surgeon. Based on the estimated incidence of CTEPH and with a better education of patients and healthcare providers, despite the advent of new interventional and medical therapies for CTEPH, the number of PEA surgeries performed should still have the potential to grow significantly.
\end{abstract}

Keywords: Chronic thromboembolic pulmonary hypertension (CTEPH); pulmonary endarterectomy (PEA); diagnosis; operability; patient selection

Submitted Aug 13, 2021. Accepted for publication Oct 08, 2021.

doi: 10.21037/acs-2021-pte-12

View this article at: https://dx.doi.org/10.21037/acs-2021-pte-12

\section{Introduction}

Chronic thromboembolic pulmonary hypertension (CTEPH) is diagnosed in $\sim 3.2 \%$ of acute pulmonary embolism (PE) survivors and results from incomplete resolution of pulmonary thromboemboli and formation of a chronic, fibrotic, flow-limiting organized thrombus within the pulmonary vascular bed $(1,2)$. The narrowing and occlusion of proximal pulmonary arteries, in combination 
with a secondary microvasculopathy of vessels less than $500 \mu \mathrm{m}$, leads to increased pulmonary vascular resistance (PVR) and progressive right ventricular (RV) failure (3). CTEPH patients typically present with progressive exercise intolerance and/or exertional dyspnea, which physiologically appear to be related to a limitation in cardiac output as well as increased dead space ventilation (4). Physical examination findings early in the course of the disease may be subtle and involve little more than an accentuated second heart sound with a prominent pulmonic component. As the disease progresses and RV dysfunction worsens, lower extremity swelling, abdominal distention, early satiety, chest pain or pressure and exertional light-headedness, with or without syncope, may also present. Hemoptysis may occur and is likely related to bronchial artery collateral circulation. With disease progression, a murmur of tricuspid regurgitation, an RV lift or gallop, fixed splitting of S2, elevated jugular venous pressure, hepatojugular reflux, ascites, hepatomegaly and peripheral edema may be noted (5). Left untreated, patients will develop severe functional limitations and will have a poor quality of life and survival (6-8).

The development of the surgical technique to perform a pulmonary endarterectomy (PEA), often also referred to as pulmonary thromboendarterectomy (PTE), along with the availability of cardiac catheterization, contrast angiography and cardiopulmonary bypass in the second half of the twentieth century, have made PEA a potentially curative treatment for CTEPH. Recently, a growing number of patients has been identified as having persistent perfusion defects resulting in persistent dyspnea after PE in the absence of pulmonary hypertension (PH). The symptoms of these patients with chronic thromboembolic pulmonary disease (CTEPD) without $\mathrm{PH}$ at rest may be associated with dead space ventilation, $\mathrm{PH}$ with exercise, and/or maladaptation of the RV and, as such, they may also benefit from PEA $(9,10)$. In this keynote lecture we will discuss the diagnosis, operability assessment and patient selection for PEA.

\section{Diagnosis}

Over the years, there have been numerous and evolving versions of diagnostic algorithms for the evaluation of CTEPH $(11,12)$. Taking into account the social demand for value-based healthcare, day-to-day clinical practice and the most recent technological developments in imaging, we will subsequently propose a diagnostic and treatment algorithm based on our most current insights. However, in addition to the question of how we diagnose CTEPH, tackling the diagnostic delay and the massive underdiagnosis are matters that deserve our equal attention.

The massive underdiagnosis of CTEPD, with or without PH, has already been described by Houk et al. in 1963 (13). They found 240 cases of chronic thromboembolic obstruction of the major pulmonary arteries, of which only six cases were correctly diagnosed before death. Thirty years later, an autopsy analysis of 13,216 patients in Poland revealed pulmonary thrombo-emboli in $5.5 \%$ of the autopsies and in up to $31.3 \%$ in the elderly population (14). If we take into account, the following numbers:

* PE incidence of 1/1,000 inhabitant/year (15);

* CTEPH incidence post PE 3\% (1);

* $\quad 57 \%$ already have CTEPH at initial presentation with $\mathrm{PE}, 43 \%$ do not (16);

* $75 \%$ of CTEPH patients have a history of PE, $25 \%$ do not (17).

Then we estimate the incidence of new CTEPH on $1,000 \times 3 \% \times 43 \% \times 100 / 75=17 /$ million inhabitants/year. This is about three times higher than the observed incidence of $5-6 /$ million inhabitants/year in registry data $(18,19)$.

Since the natural course of CTEPH involves progressive remodeling of the distal arteries and increase of PVR, which are both important determinants of outcome, early CTEPH diagnosis and referral to a CTEPH center seem to be critical for optimal treatment. Indeed, Klok et al. recently demonstrated that CTEPH patients with longer diagnostic delay had a less favorable hemodynamic profile and shorter survival, possibly reflecting more severe concomitant secondary vascular disease and poorer RV function (20). However, early diagnosis of CTEPH remains a major challenge, as demonstrated by a median delay of fourteen months between the time of clinical presentation and diagnosis identified in the International CTEPH Registry (17).

Both the underdiagnosis and diagnostic delay might be related to various causes: (I) the often nonspecific clinical presentation of the disease and, early in its natural history, its subtle physical examination findings; (II) failure to consider disorders of the pulmonary vascular bed in patients with unexplained dyspnea and as such, diagnostic misclassifications as acute PE or other conditions; (III) a tendency to discount the possibility of CTEPD in the absence of a documented history of acute venous thromboembolism; (IV) a lack of awareness of the disease entity by many physicians and; (V) the cumbersome diagnostic process of CTEPH, which involves 


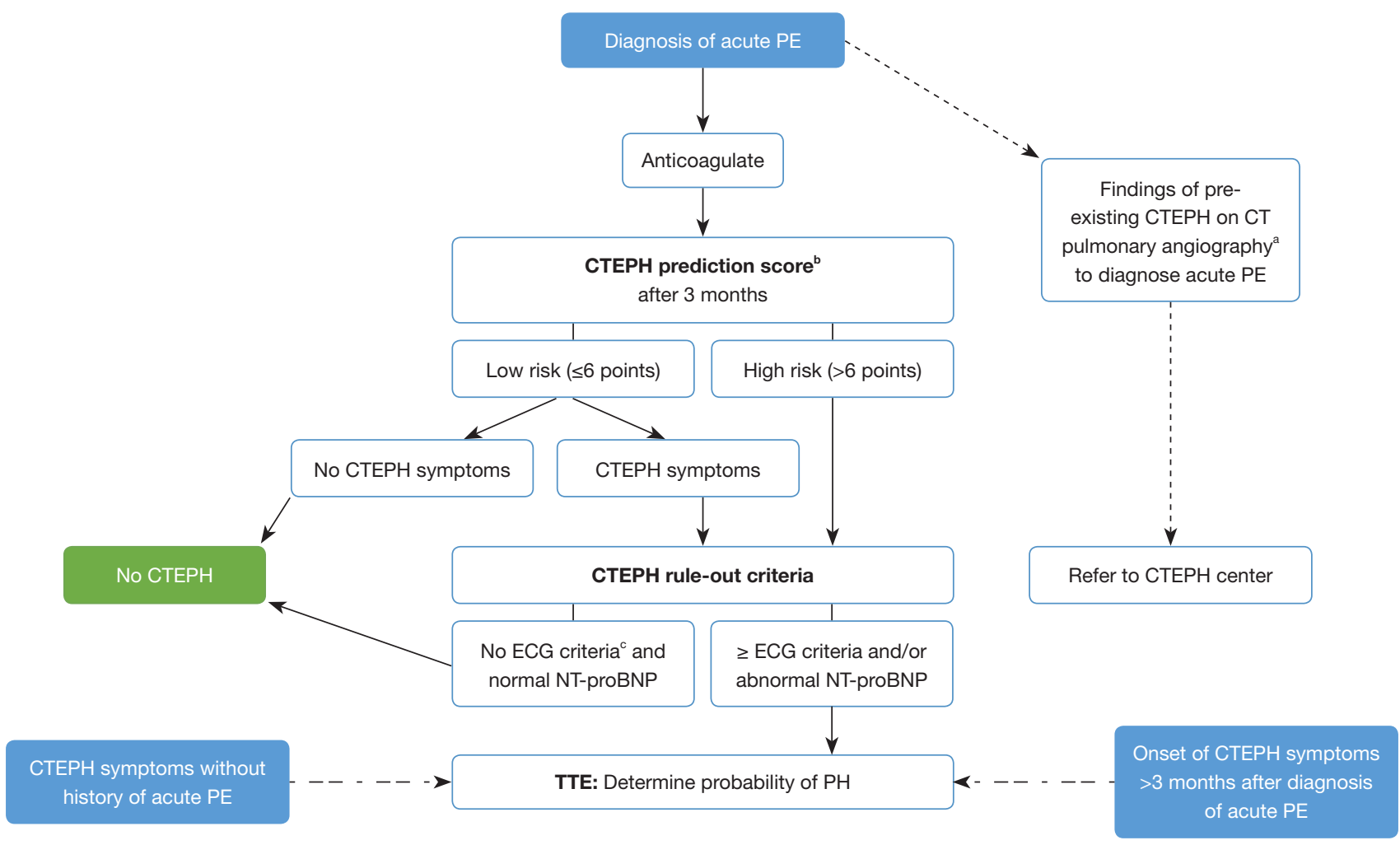

Figure 1 Screening algorithm for CTEPH. ${ }^{\text {a, b, }}$, see tables and figure below. PE, pulmonary embolism; CTEPH, chronic thromboembolic pulmonary hypertension; PH, pulmonary hypertension; ECG, electrocardiogram; NT-proBNP, N-terminal pro-brain natriuretic peptide; TTE, transthoracic echocardiography.

multiple healthcare providers from different clinical specialties $(21,22)$.

\section{Screening after acute $\mathrm{PE}$}

Education of physicians about CTEPH to tackle the underdiagnosis and diagnostic delay is paramount and is the responsibility of all members of the CTEPH multidisciplinary team. In addition, subjecting all acute $\mathrm{PE}$ survivors to diagnostic testing for CTEPH seems to be the next logical choice to arrive at a faster diagnosis. Thrombus resolution is mostly achieved at three months, therefore, it seems logical to perform diagnostic tests at this time point. It additionally coincides with the time of routine evaluation of the duration and intensity of anticoagulant treatment and, the evaluation of presence of cancer and/or systemic cardiovascular disease (12). Also, physical recovery from acute PE should be achieved by this time-point. Transthoracic echocardiography (TTE) is the recommended screening tool for suspected PH (11).
However, subjecting all acute PE survivors to TTE has been shown to have a low diagnostic yield, to result in overdiagnosis and is cost-ineffective (23). The 2019 European Guidelines on PE recommend applying TTE three to six months after PE diagnosis in all patients with persistent dyspnea and/or predisposing conditions for CTEPH (12). However, only 3.2\% of acute PE survivors are thought to develop CTEPH however, $50 \%$ of patients with PE report persistent dyspnea to some degree (24). As such, a considerable number of patients will require TTE according to this guideline, although sufficient resources may not be available globally.

We therefore, propose in Figure 1 to apply a dedicated CTEPH screening algorithm three months after the diagnosis of an acute PE, based on the InShape II study results (25). Pretest probability of CTEPH is assessed by calculating the 'CTEPH prediction score' (Table 1) (26). Only patients with $>6$ points or those with symptoms that might be associated with CTEPH (i.e., exertional dyspnea, edema, newly developed palpitations, syncope 


\begin{tabular}{|c|c|}
\hline & Score \\
\hline Unprovoked PE & +6 points \\
\hline Known hypothyroidism & +3 points \\
\hline Symptom onset $<2$ weeks before PE diagnosis & +3 points \\
\hline Right ventricular dysfunction on $\mathrm{CT}$ or TTE & +2 points \\
\hline Known diabetes mellitus & -3 points \\
\hline $\begin{array}{l}\text { Thrombolytic therapy or embolectomy for the } \\
\text { acute PE event }\end{array}$ & -3 points \\
\hline \multicolumn{2}{|c|}{$\begin{array}{l}\text { Low risk: } \leq 6 \text { points. High risk: }>6 \text { points. CTEPH, chronic } \\
\text { thromboembolic pulmonary hypertension; PE, pulmonary } \\
\text { embolism; CT, computed tomography, TTE, transthoracic } \\
\text { echocardiography. }\end{array}$} \\
\hline
\end{tabular}

or chest pain) are subjected to the CTEPH rule-out criteria, that is, assessment of the presence of any of the three ECG criteria of RV pressure overload (Figure 2), or an abnormal age-dependent and gender-dependent NT-proBNP level (25). If one of these rule-out criteria is present, the patient is referred for a TTE. The InShape II study demonstrated that this screening algorithm accurately and early excluded CTEPH after acute PE, while avoiding TTE in $81 \%$ of patients. Moreover, the vast majority of CTEPH diagnoses were made within four months of the index PE, which is substantially earlier than the fourteenmonth diagnostic delay, for example, which is reported in current clinical practice $(17,25)$. However, both this screening tool and TTE may not identify early disease stages (25). Therefore, and also taking into account the consideration of many CTEPH centers to operate on selected cases of CTEPD without $\mathrm{PH}$, we propose to skip the screening step and to directly refer the patient to a CTEPH center if findings of pre-existing CTEPD are present on the computed tomography pulmonary angiography (CTPA) performed to diagnose the acute $\mathrm{PE}$ (Figure 1). These CTPA findings are listed in Table 2.

\section{Late presentation or no history of acute PE}

Still two important groups of patients that are finally diagnosed with CTEPH remain: (I) patients that develop CTEPH $>3$ months after the diagnosis of acute PE and hence are not guided towards a TTE by the screening tool and; (II) patients that develop CTEPH symptoms without history of acute PE. This group might compromise about
$25 \%$ of all CTEPH patients (17). Both groups are therefore, also added on our screening algorithm in Figure 1. The first group emphasizes the importance of remaining vigilant for CTEPH if new-onset dyspnea develops years after an acute $\mathrm{PE}$ diagnosis, independent of diagnostic tests shortly after the PE. The second group emphasizes the importance of physicians being aware of the existence of the disease. Most physicians will refer these patients, based on their clinical presentation, for TTE. But the challenge lies in educating cardiologists to screen for PH on TTE and, if present in absence of left-sided valvular pathology and without history of acute $\mathrm{PE}$, to refer for ventilation/perfusion scintigraphy (V/Q scan)

\section{TTE}

The diagnostic work-up of suspected CTEPH is depicted in Figure 3 and starts with transthoracic Doppler echocardiography. Echocardiographic signs suggesting $\mathrm{PH}$ are shown in Table 3 (11). In patients without symptoms, a low echocardiographic probability result should rule out CTEPH. However, TTE follow-up may be considered in low probability patients with otherwise unexplained dyspnea (27). Patients with an intermediate echocardiographic probability and an elevated NT-proBNP and/or risk factors for CTEPH (see Table 4) and/or abnormal cardiopulmonary exercise testing (CPET) results and, patients with high echocardiographic $\mathrm{PH}$ probability, should undergo a V/Q scan (11). As TTE may not identify early disease stages and CPET has been demonstrated to be able to detect CTEPH despite normal TTE $(25,28)$, we propose to also consider these 3 factors for low probability but symptomatic patients. Stress echocardiography is also able to identify patients with disproportionate increase in pulmonary arterial pressure (PAP) at exercise, but the prognostic implications of exercise-induced $\mathrm{PH}$ in these patients requires prospective validation (29). However, PH is also not uncommon in the setting of left heart disease and its presence even serves as an indication for valve surgery. Therefore, left heart disease should be ruled out before performing a V/Q scan (30).

\section{Ventilation/perfusion scintigraphy (V/Q scan)}

V/Q scanning is highly sensitive (96\% to $97.4 \%$ ) in detecting perfusion abnormalities and with a negative predictive value of nearly $100 \%$, a normal V/Q scan excludes the diagnosis of CTEPH (31). It is therefore 

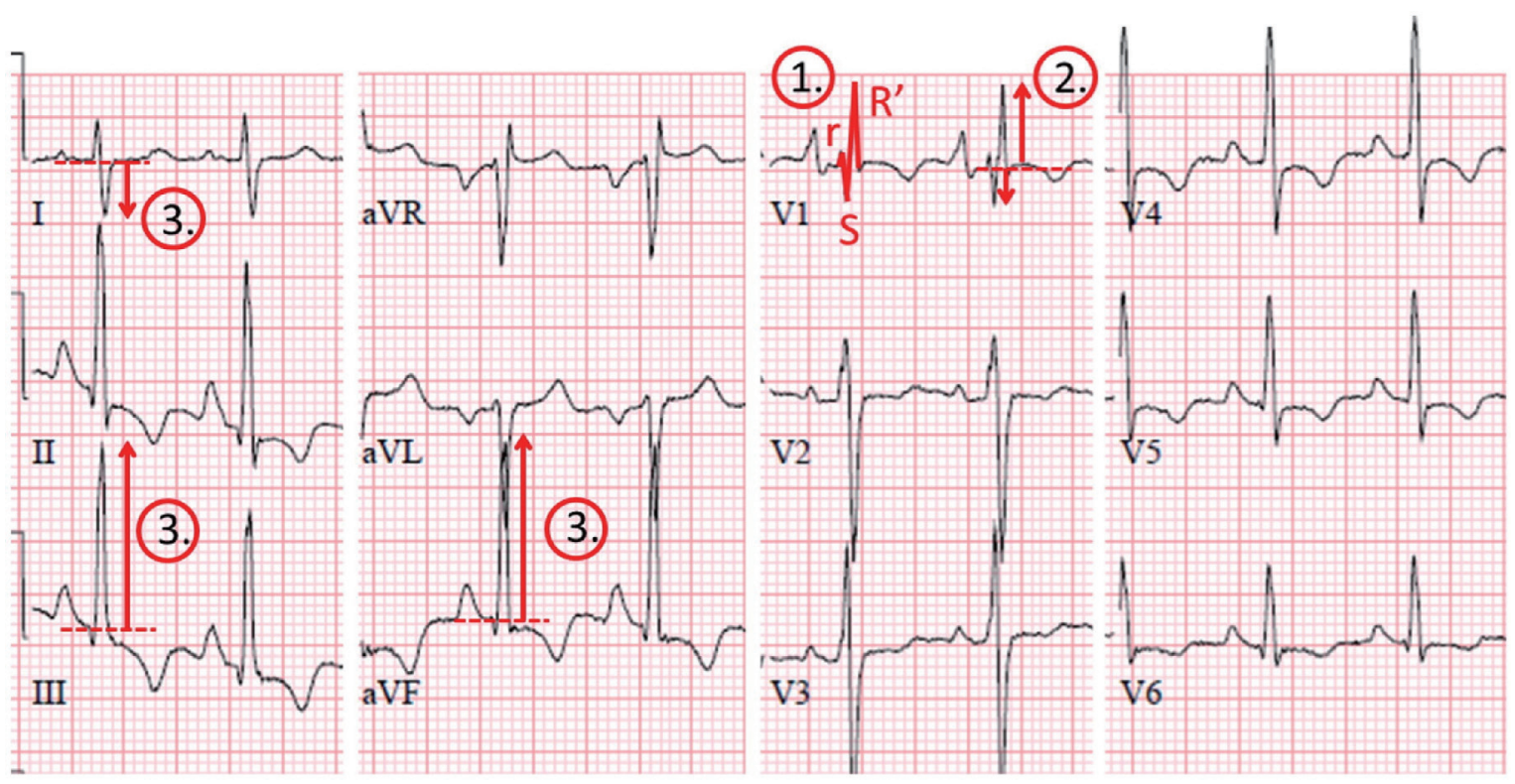

Figure 2 ECG demonstrating the three specific electrocardiographic characteristics of right ventricular overload, the 'Chronic Thromboembolic Pulmonary Hypertension (CTEPH) rule out criteria'. (1) Right bundle branch block: rSR' of RSr' pattern in lead V1 with a QRS duration $\geq 120 \mathrm{~ms}$; (2) R:S $>1$ in lead V1 with $\mathrm{R}>0.5 \mathrm{mV}$ and (3) right QRS axis deviation QRS axis $>90^{\circ}$. Reprinted from "To screen or not to screen for chronic thromboembolic pulmonary hypertension after acute pulmonary embolism" (26), Vol 151, Yvonne M. EndeVerhaar, Menno V. Huisman, Frederikus A. Klok, Pages No.7, Copyright [2017], with permission from Elsevier. ECG, electrocardiography.

Table 2 Findings of pre-existing CTEPH on computed tomography pulmonary angiography

Direct vascular signs

Eccentric wall-adherent filling defect(s), which may calcify; different from the central filling defects within a distended lumen, which are the hallmark of acute PE

Abrupt tapering and truncation

Complete occlusion and pouch defects

Intimal irregularity

Linear intraluminal filling defects (intravascular webs and bands)

Stenosis and post-stenotic dilatation

Vascular tortuosity

Indirect vascular signs

Significant RV hypertrophy, RA dilatation

Pericardial effusion

Dilatation of pulmonary artery ( $>29 \mathrm{~mm}$ in men and $>27 \mathrm{~mm}$ in women) and/or calcifications of pulmonary artery

Systemic collateral arterial supply (bronchial arterial collaterals towards pulmonary post-obstructive vessels)

Parenchymal changes

Mosaic attenuation of the lung parenchyma resulting in geographical variation in perfusion

Data from $(12,27)$. CTEPH, chronic thromboembolic pulmonary hypertension; PE, pulmonary embolism; RV, right ventricular; RA, right atrial. 


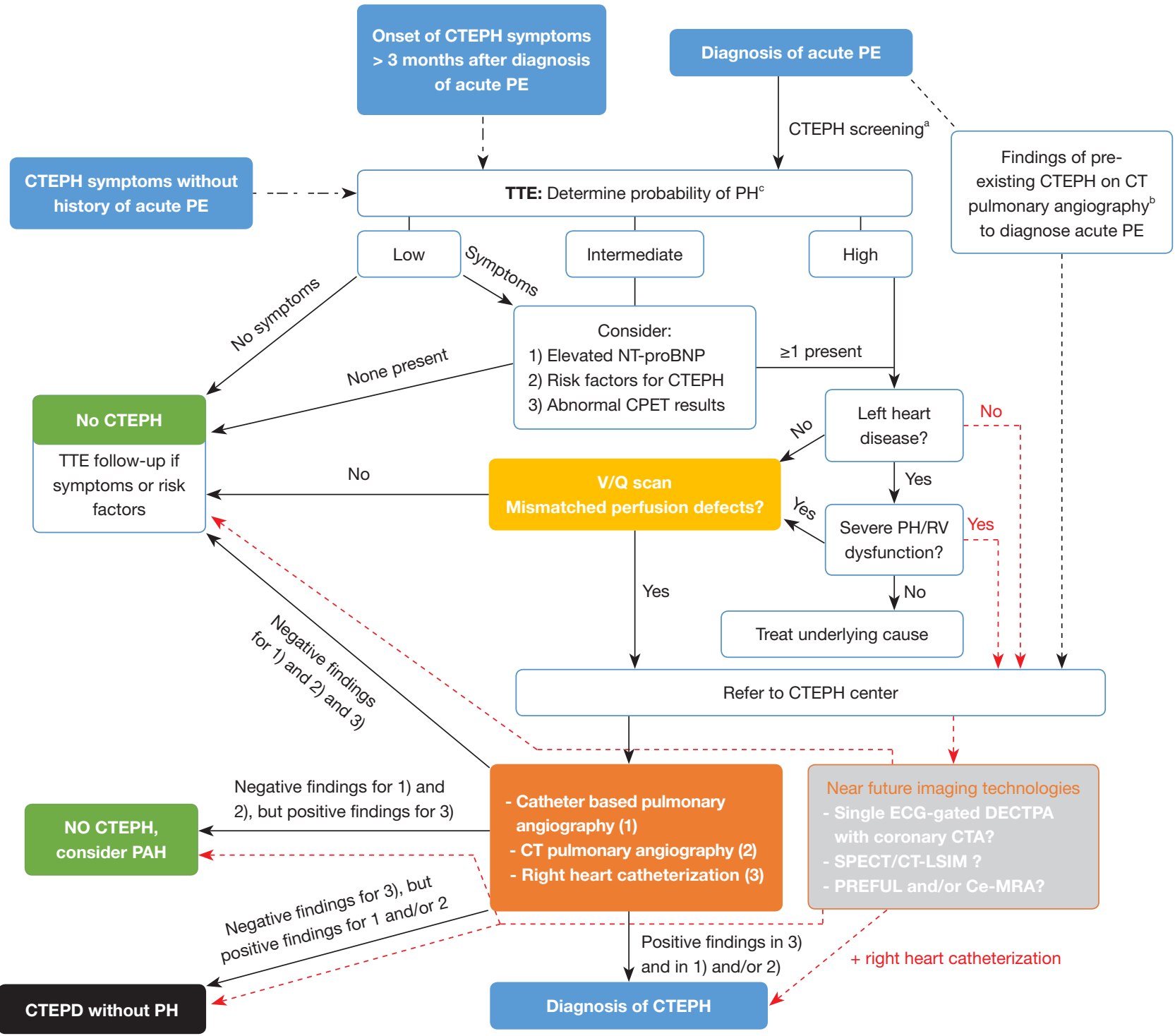

Figure 3 Diagnostic algorithm for CTEPH. ${ }^{\text {a, b, c }}$, see tables and figure below. CTEPH, chronic thromboembolic pulmonary hypertension; PE, pulmonary embolism; CTEPD, chronic thromboembolic pulmonary disease; TTE, transthoracic echocardiography; PH, pulmonary hypertension; NT-proBNP, N-terminal pro-brain natriuretic peptide; CPET, cardiopulmonary exercise testing; V/Q, ventilation/perfusion; RV, right ventricular; CT, computed tomography; PAH, pulmonary arterial hypertension; ECG, electrocardiogram; DECTPA, dual-energy computed tomography pulmonary angiography; CTA, computed tomography angiography; SPECT, Single-photon emission computed tomography; CT-LSIM, computed tomography lung subtraction iodine mapping; Ce-MRA, contrast enhanced magnetic resonance angiography; PREFUL, phase-resolved functional lung.

currently considered the "gold standard" screening method for CTEPH (Figure 4A). However, the non-specificity of this modality requires additional diagnostic imaging in case of mismatched perfusion deficits to definitively diagnose CTEPH.

\section{Catheter-based pulmonary angiography and right heart catheterization}

Catheter-based pulmonary angiography has been considered the "gold standard" for imaging in the 


\begin{tabular}{lll}
\multicolumn{2}{l}{ Table 3 Echocardiographic probability of $\mathrm{PH}$ in symptomatic patients with a suspicion of $\mathrm{PH}$} & \\
\hline Peak tricuspid regurgitation velocity $\mathrm{m} / \mathrm{s}$ & Presence of other echocardiographic signs of $\mathrm{PH}^{\mathrm{a}}$ & Echocardiographic probability of PH \\
\hline$\leq 2.8$ or not measurable & No & Low \\
$\leq 2.8$ or not measurable & Yes & Intermediate \\
$2.9-3.4$ & No & High \\
$2.9-3.4$ & Yes & \\
$>3.4$ & Not required &
\end{tabular}

${ }^{\text {a }}$, right ventricle/left ventricle basal diameter ratio $>1.0$, flattening of the interventricular septum (left ventricular eccentricity index $>1.1$ in systole and/or diastole), right ventricular outflow doppler acceleration time $<105 \mathrm{~ms}$ and/or mid-systolic notching, early diastolic pulmonary regurgitation velocity $<2.2 \mathrm{~m} / \mathrm{s}$, pulmonary artery diameter $>25 \mathrm{~mm}$, inferior cava diameter $>21 \mathrm{~mm}$ with decreased inspiratory collapse ( $<50 \%$ with a sniff of $<20 \%$ with quiet inspiration), right atrial area (end-systole) $>18 \mathrm{~cm}^{2}$. Reproduced from (11) with permission. $\mathrm{PH}$, pulmonary hypertension.

Table 4 Risk factors and predisposing conditions for CTEPH

\begin{tabular}{|c|c|}
\hline Findings related to the acute PE event ${ }^{a}$ & Concomitant chronic diseases and conditions predisposing to $\mathrm{CTEPH}^{\mathrm{C}}$ \\
\hline Large pulmonary arterial thrombi on CTPA & Infected chronic i.v. lines or pacemakers \\
\hline Echocardiographic signs of $\mathrm{PH} / \mathrm{RV}$ dysfunction & History of splenectomy \\
\hline \multirow[t]{4}{*}{ CTPA findings suggestive of CTEPD ${ }^{b}$} & $\begin{array}{l}\text { Thrombophilic disorders, particularly antiphospholipid antibody syndrome and high } \\
\text { coagulation factor VIII levels }\end{array}$ \\
\hline & Hypothyroidism treated with thyroid hormones \\
\hline & History of cancer \\
\hline & Myeloproliferative disorders \\
\hline
\end{tabular}

evaluation of CTEPH. When combined with right heart catherization, it can confirm the presence of CTEPH to the level of subsegmental vessels, exclude other possible diagnoses, accurately localize or "map out" lesions in the determination of surgical accessibility, and evaluate pulmonary hemodynamics and right heart function $(2,32)$. Characteristic pulmonary angiographic findings suggestive of CTEPH include webs or bands, intimal irregularities, pouch defects, abrupt vascular narrowing, and complete obstruction of pulmonary arteries (Figure 4B) (32).

\section{CTPA}

CTPA can be used to assess operability as it can provide detailed structural information including endovascular thrombi, vascular wall thickness, intraluminal fibrous bands or webs, stenosis and bronchial artery collateral circulation (Figure 4C) (30). It may also reveal a mosaic perfusion pattern of the pulmonary parenchyma, suggesting CTEPH. Additional benefits are the assessment of possible underlying parenchymal lung and mediastinal disease and 
the detection of other pulmonary vessel disorders that may present with perfusion defects on V/Q lung scanning such as pulmonary artery sarcoma, pulmonary veno-occlusive disease or fibrosing mediastinitis (2). However, difficulties with this imaging modality relate to the interpretive expertise required and the lower sensitivity for detecting CTEPD in segmental and subsegmental vessels $(33,34)$.

Next to right heart catheterization to obtain the hemodynamic confirmation of CTEPH, as presented in Figure 3, many CTEPH centers have in the last decade used a combination of catheter based pulmonary angiography and CTPA to obtain the benefits of both imaging techniques to optimally diagnose and assess the operability of CTEPH. Elevated PAP on right heart catheterization with negative findings on pulmonary angiography and CTPA, exclude CTEPH, but not other forms of PH (for example, pulmonary arterial hypertension). On the other hand, normal PAP on right heart catheterization but positive findings on pulmonary angiography and/or CTPA may indicate CTEPD, without PH. Figure 4 illustrates these different imaging modalities for a single patient. This traditional imaging workup has however some inherent disadvantages:

(I) With V/Q 2D images, segmental defects may be missed or underestimated due to segmental overlap and shine-through masking of adjacent lung at certain camera positions (35).

(II) A positive V/Q scan can be confounded by other etiologies of pulmonary malperfusion and does not provide structural information to guide operability assessment. Therefore, additional diagnostic imaging is paramount to definitively diagnose CTEPH.

(III) The combined advantages and disadvantages of pulmonary angiography and CTPA prevent the use of only one of the two techniques to achieve a reliable diagnosis and assessment of operability.

(IV) As such, the cumulative radiation and contrast exposure and costs of a V/Q scan, digital subtraction pulmonary angiography, CTPA, and coronary artery catheterization are high (36).

Advances in imaging techniques in the last decade try to address these issues. Single-photon emission computed tomography (SPECT) cameras provide higher sensitivity $3 \mathrm{D}$ images that overcome the limitations of V/Q 2D scanning (37). Moreover, these V/Q SPECT images can be fused with CTPA images to provide further structural and functional information (35). ECG-gated CT can provide detailed visualization of segmental and subsegmental vessels, lung parenchyma and RV myocardial size and morphology and allows quantitative assessment of RV function with lower radiation dose than conventional CTPA $(38,39)$. It can also be used to assess for significant coronary artery disease, which needs to be excluded when working up patients for PEA (38). Also, dual-energy CT (DECT) increases the sensitivity and specificity of the diagnosis of CTEPH $(30,40)$. Moreover, it can also serve to assess pulmonary perfusion in patients with CTEPH by calculating perfused blood volume maps from iodine distribution in the lung parenchyma (41). The merging of anatomical and physiologic data with DECT angiography improves the detection of distal CTEPH and provides more accurate information on overall pulmonary vascular reserve and parenchymal arterial perfusion (42).

MRI has emerged as the reference standard in the assessment of RV size and systolic function and contrastenhanced MR angiography (ce-MRA) appears to be superior to pulmonary angiography in depicting the precise proximal beginning of the thromboembolic material, in good correlation with the origin of the dissection plane during PEA (43). Additionally, Phase-Resolved Functional Lung (PREFUL)-MRI could detect and quantify hemodynamic changes in CTEPH. The initial results of this technique are promising, considering no intravenous contrast is required (44). Therefore, a combination of CTPA for morphological delineation and MRI for functional and pulmonary vascular assessment with pulmonary angiography reserved as a problem-solving tool has been proposed in previous diagnostic algorithms. However, MRI remains an expensive examination with limited availability and therefore difficulties in didactic implementation due to a lack of appropriate equipment and/or expertise. Moreover, recently the accuracy of ECG-gated CT angiography in assessing pulmonary arteries for signs of CTEPH has been reported to be superior to both ce-MRA and pulmonary angiography (34).

A novel approach whereby one imaging study is performed that can provide qualitative and quantitative assessment of pulmonary perfusion, high spatial resolution assessment of the pulmonary arteries and coronary arteries, and morphologic and quantitative assessment of the heart, all with lower radiation exposure and overall cost, is a great desire of every multidisciplinary CTEPH team. A single ECG-gated dual energy CTPA and coronary CT angiography exam was recently proposed by Kligerman and Hsiao as such a strategy (36). Although this strategy provides a promising single imaging study for screening 
A B
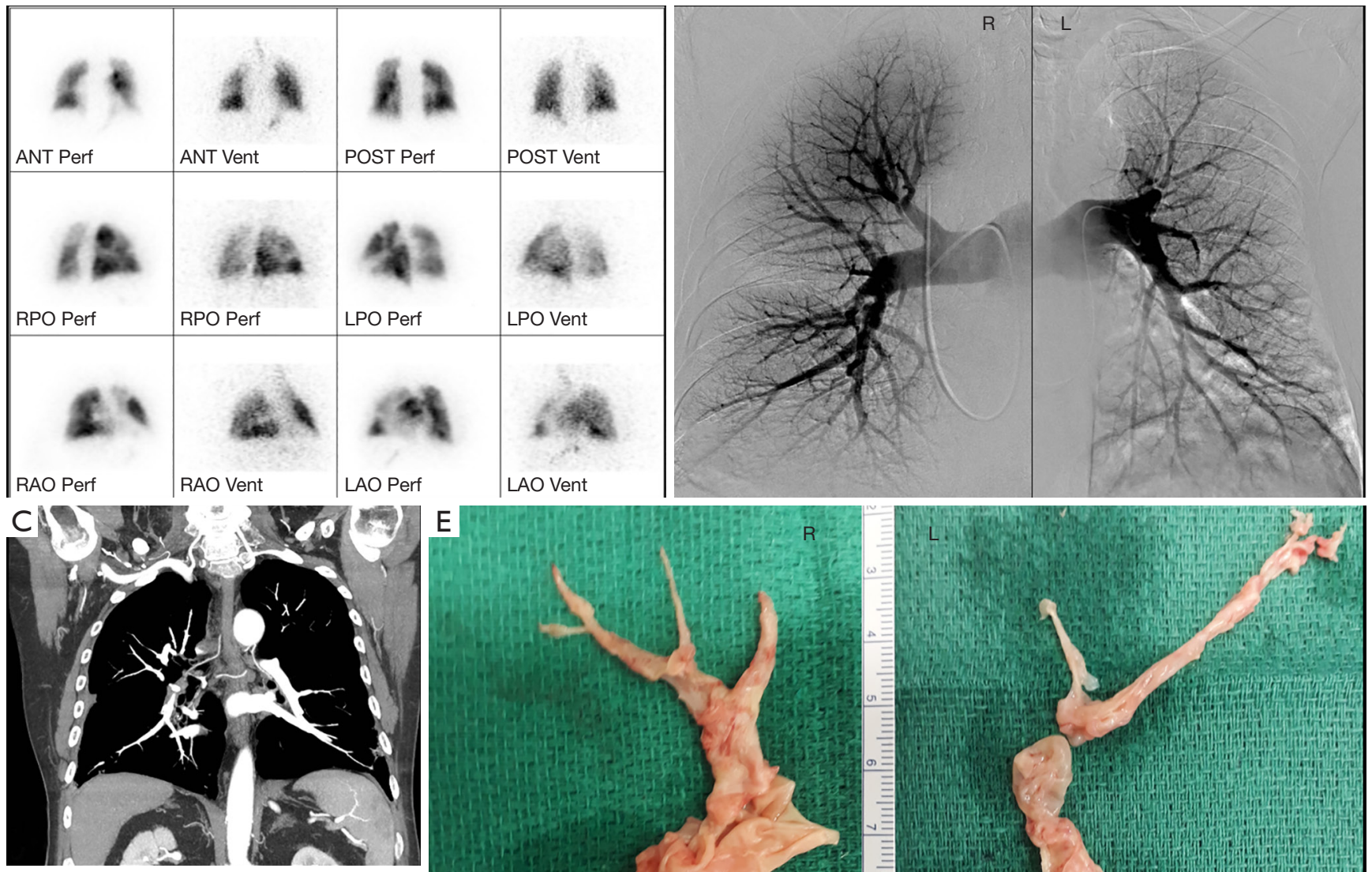

E
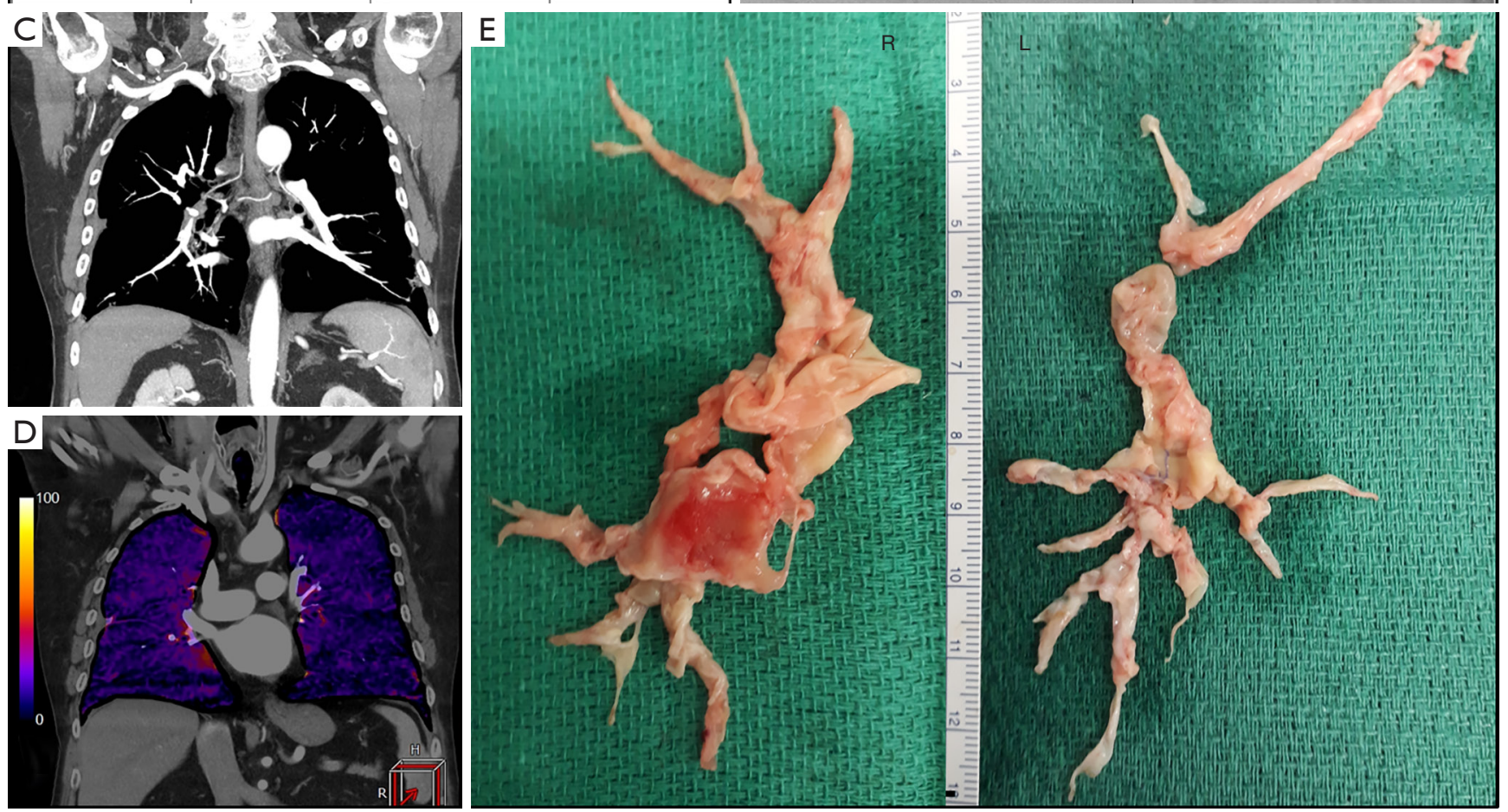

Figure 4 Imaging modalities and resection specimens from a single patient. (A) V/Q scan showing decreased perfusion with preserved ventilation in the posterior left upper lobe, the lateral left upper lobe, the lateral left lower lobe and small perfusion deficits in the right middle lobe. (B) Digital subtraction pulmonary angiography showing (R): multiple stenoses in the proximal segmental arteries of the lower lobe arteries (most pronounced basal-posterior), in the posterior segmental artery of the upper lobe and in the bifurcation of the upper lobe artery that bifurcates in the segmental branches to the apical and anterior segment. Wall-mounted thrombus material in upper lobar artery and in the posterior segmental artery of the upper lobe. Truncation of some arteries in the middle lobe. (L): stenosis proximal in the upper lobe artery with slight irregular outline of het proximal upper lobe artery. Proximal caliber variations in multiple segmental lower lobe arteries. Slight irregular outline van some lingular branches, with truncations of some of these branches. Truncation of some basal branches in the lower lobe. (C) Coronal MIP reconstruction of CTPA during late arterial phase, visualizing chronic PE and bronchial arterial hypertrophy. (D) Coronal iodine map imaging (CT-LSIM) demonstrating severe patchy perfusion defects. (E) Surgical resection specimens. $\mathrm{R}$, right; L, left; MIP, maximum intension projection; CTPA, computed tomography pulmonary angiography; PE, pulmonary embolism; CT-LSIM, computed tomography lung subtraction iodine mapping. 
and operability assessment with lower radiation exposure and costs than current practice, it is not yet researched thoroughly nor validated for this purpose. Moreover, also other emerging imaging techniques may be considered in combination with a coronary CT angiography exam. As such, the recently published Pulmonary Vascular Research Institute (PVRI) Diagnostic algorithm for PH includes CTlung subtraction iodine mapping (CT-LSIM) (Figure 4D), SPECT and/or MRI perfusion (30).

Therefore, given we are convinced that new developments in CT and MR imaging might definitively change the diagnostic work-up, we included a potential future pathway in our schematic overview of the diagnostic algorithm of CTEPH (see red dotted lines in Figure 3). Taking into account the desire to require only one imaging test for the diagnosis and assessment of the operability of CTEPH and the expected requirement of a time-consuming training and a high level of expertise among radiologists, we propose to directly refer high $\mathrm{PH}$ probability patients without left heart disease to a CTEPH center to undergo a diagnostic work-up with these new imaging technologies. One should however also realize that a global implementation of these emerging imaging techniques may be limited by reduced financial resources and access to these techniques, and that all these techniques need more research and validation before they could potentially replace the traditional imaging approach to CTEPH.

\section{Operability assessment and patient selection}

PEA is the recommended treatment for patients with CTEPH who are good surgical candidates. Determining surgical candidacy involves two separate processes: (I) an evaluation of technical operability and; (II) an assessment of the potential risks and benefits of surgery (see Figure 5) which should be performed by an experienced multidisciplinary CTEPH team, including at least one experienced surgeon $(11,45)$.

Technical operability ultimately depends on both the anatomic location of the CTEPD and the skill and experience of the surgeon. The patient must have sufficient surgically accessible thromboembolic material, with a proportional PVR indicating the absence of extensive secondary vasculopathy (17). The intraoperative classification of the disease is based on the most central component of the disease. Level 0 defines no evidence of thromboembolic disease in either lung; level I defines disease involving the main pulmonary arteries (with level
IC defining complete occlusion of one main pulmonary artery); level II the lobar branches; level III the segmental branches and; level IV the subsegmental arteries (46). Surgical clearing of disease in the main, lobar and proximal segmental pulmonary artery branches (level I-III) should be feasible by any skilled and properly trained PEA surgeon. The CHEST-1 study provided a definition of an experienced PEA surgeon, as one who has performed $>20$ PEAs in the year they started to assess study cases, and/or $>20$ in the year before they started to assess study cases, and/or $>40$ in the three years before they started to assess study cases (47). However, distal segmental and subsegmental disease (level III-IV) is more difficult to remove and the technical feasibility might depend more on the surgeon's experience (volume load/year) than on his/ her skills. Criteria have also been proposed to classify the level of expertise of a CTEPH center: surgical mortality $<5 \%$ (level I) and surgical volume defined as $\geq 50$ PEA's/year (level II) and the ability to operate on distal disease and the ability to provide PEA, balloon pulmonary angioplasty (BPA) and medical therapy (level III) (8). In our opinion these definitions and criteria are justified with the sole purpose of offering the best possible treatment strategies available to all CTEPH patients. Despite the fact that the number of PEAs performed per center could not predict improved long-term survival on multivariable analysis in the international CTEPH registry, a clear trend was demonstrated for in-hospital mortality to lower when the number of PEA's performed per year in a center were higher $(7,48)$. It was therefore previously suggested that there should be only one experienced CTEPH center per forty to fifty million population performing no fewer than fifty cases per year and with in-hospital mortality rates $<5 \%(8)$.

Despite having excellent healthcare systems, many smaller countries have populations well below the forty to fifty million mark. At least organizing the care for CTEPH by national governments and health systems in a single center in these countries therefore seems imperative. Even merging CTEPH centers across borders could be suggested, but inevitably encounters problems related to the difference in health care systems between different countries. However, growing institutional experience decreases mortality rate (49). This also results in in-hospital mortality rates reported by CTEPH centers from smaller countries well below 5\% (for example, Leuven 2016-2020: $3.8 \%$, unpublished results) $(17,50)$. However, despite qualitative care, as a result of years of experience of the 


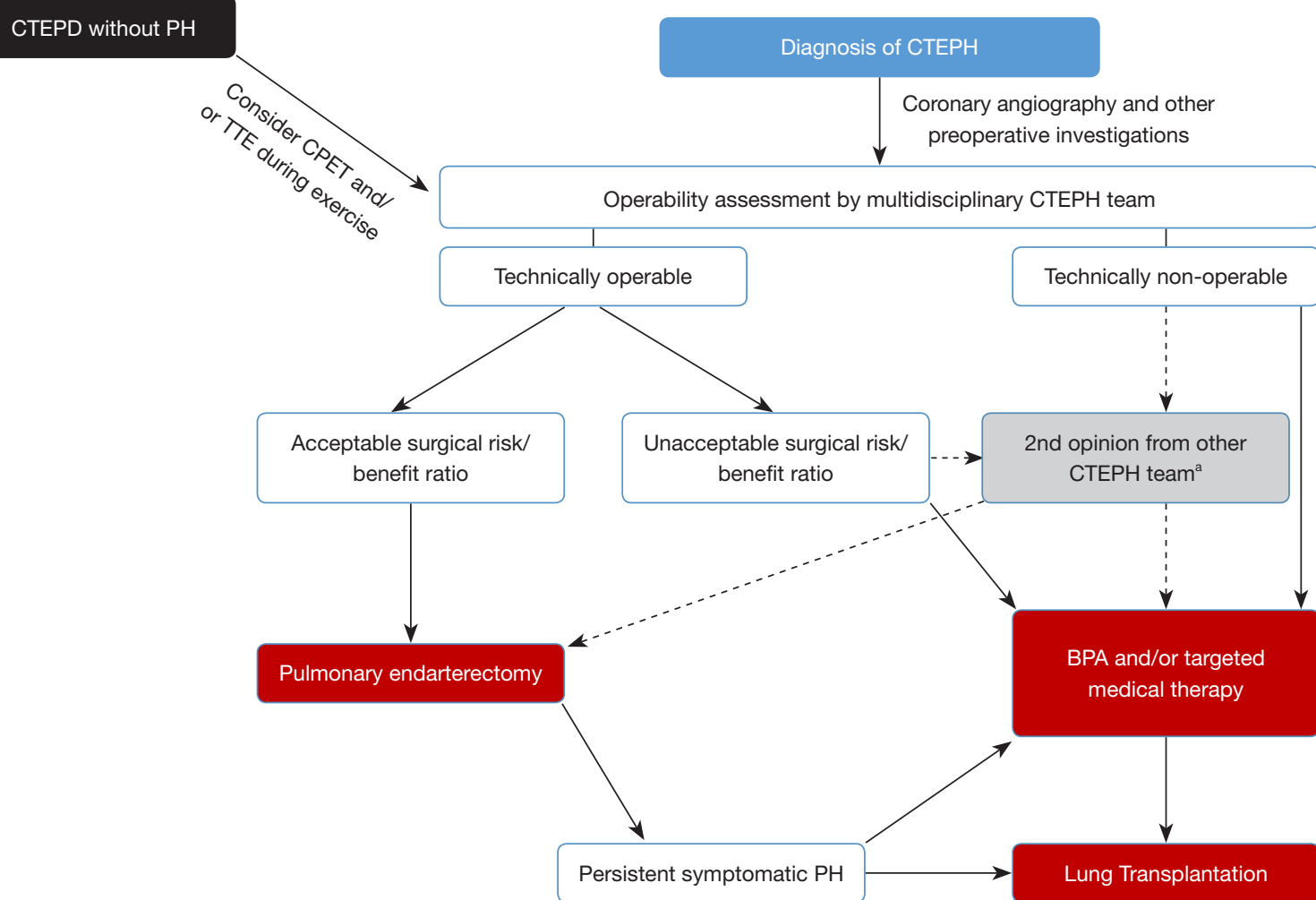

Figure 5 Operability assessment by multidisciplinary CTEPH team. a , for smaller centers/countries. Adapted from (11) with permission. CTEPD, chronic thromboembolic pulmonary disease; PH, pulmonary hypertension; CTEPH, chronic thromboembolic pulmonary hypertension; CPET, cardiopulmonary exercise testing; TTE, transthoracic echocardiography; BPA, balloon pulmonary angioplasty; PH, pulmonary hypertension.

whole CTEPH team in these centers, the experience with surgery for level IV disease and as such the assessment of technical operability remains most probably different than in larger volume centers. This was clearly demonstrated in the International prospective CTEPH registry as lowvolume centers reported significantly higher percentages of non-operable patients compared to high volume centers, suggesting that center expertise may have influenced the decision to operate (17). Therefore, a second surgical opinion from a large volume CTEPH center and even referral to such a center may be recommended for the surgical treatment of level IV disease (45). On the other hand, the treatment landscape of CTEPH is changing and BPA has emerged as a valuable treatment option for nonoperable disease. Many CTEPH centers, also in smaller countries, have started BPA programs. Undoubtedly, in many studies the assessment of non-operability as justification to perform a BPA has included anatomical level IV and even level III, and the results of BPA in these settings were at least comparable to the PEA results (51). Therefore, the ability of a center to perform PEA for level IV disease might become a less strict criterion to be considered as an expert or high-quality center.

As depicted in Figure 5, if the patient is assessed as technically operable, the next step is to assess the risk/ benefit ratio. The potential likelihood of hemodynamic and symptomatic improvement is an important consideration to argue in favor of a PEA. However, symptomatic improvement largely depends on the individual patient expectations, which can be significantly different for a young patient compared to an octogenarian patient with similar symptoms. Hemodynamic improvement is dependent on the correlation of accessible surgical disease and the severity of PH and RV dysfunction (46). As such, initially, symptomatic CTEPH represented the sole indication for surgery. However, even in the absence of resting PH, patients with CTEPD may have functional limitations due to increased dead space ventilation or an abnormal 
pulmonary hemodynamic response during exercise and may benefit from PEA $(9,10,52)$. Sometimes PEA is offered to CTEPD patients without $\mathrm{PH}$, arguing it will not only improve symptoms but will also prevent potential chronic parenchymal changes, scarring and secondary vasculopathy. However, it is not yet clear whether CTEPD without PH is a precursor of CTEPH with a variable time course or a separate entity. We therefore, try to further assess the potential benefit of a PEA in these CTEPD patients by performing CPET and stress echocardiography or MRI to disclose impaired cardiac reserve and significant pulmonary vascular disease (53).

Parenchymal lung disease (severe emphysema or interstitial lung disease) is the only absolute contraindication for a PEA, as these patients will derive little benefit from a PEA because the attempted improvement in perfusion may not improve symptoms if ventilation is severely compromised, and the risk of respiratory failure after surgery is significant (46). With the exception of those that are terminal or end stage, no other comorbid conditions are absolute contra-indications to PEA. Age per se, nor obesity, are contraindications with studies demonstrating excellent results in children, octogenarians and obese patients (54-57). However, some coexisting conditions impose an increased risk on less favorable peri-operative and long-term outcomes: an absent history of deep vein thrombosis or $\mathrm{PE}$, signs of right heart failure, significant pulmonary or left heart disease [with need for concomitant cardiac surgery (7)] World Health Organization functional class IV, inconsistency on imaging modalities, absence of appreciable lower lobe disease, PVR $>1,200$ dynes.s $/ \mathrm{cm}^{5}$, out of proportion to site and number of obstruction on imaging; and higher diastolic PAP (58). These risk factors do not represent an absolute contraindication to PEA and should be weighed against the potential symptomatic benefits of PEA by the multidisciplinary CTEPH team. They should then be carefully reviewed with the patient before the decision is made to proceed to surgery (5). However, no less than $20 \%$ of operable patients seem to refuse PEA because of the perceived high risk of surgery (22). As we feel that the need for ongoing education and better understanding of CTEPH treatment outside CTEPH centers might be related to this misperception, and also contribute to the delay or lack of referral for further diagnostic work-up and treatment, we highly recommend referring patients to a CTEPH center as early as possible. This means after each TTE with high PH probability or after a V/Q scan with mismatched perfusion defects, at the latest. In order to avoid conservative or alternative treatment strategies for CTEPH patients that should have been treated with a PEA, we recommend to leave the consultation with the patient regarding the decision to surgery to a member of the CTEPH team, preferably to the performing surgeon, in order to correctly inform the patient of the low mortality rates and the possible improvement of exercise capacity and quality of life he/she might expect after a PEA. Hence, an in-hospital mortality rate of $4.7 \%$ was reported by the international CTEPH registry and even as low as $2.2 \%$ by the center with the largest experience with PEA $(17,49)$. Long-term survival as high as $89 \%$ after three years and $75 \%$ after ten years have been reported $(7,49,59)$. Moreover, for patients who refuse PEA, a five-year survival rate of $53 \%$ has been reported vs. $83 \%$ for those who underwent surgery (60).

Nonetheless, CTEPH operability assessment undoubtedly remains subjective with even disagreements among experts. In the CHEST- 1 study $22 \%$ of patients who were initially deemed inoperable by experts, upon second review were changed to operable (47). This observation should make us aware that the reported $60 \%$ of CTEPH patients deemed operable in the international CTEPH registry could have the potential to increase.

As such, it is the delay in and/or lack of referral to CTEPH centers, the subjective process of operability assessment and the refusal of surgery by sub-optimally/ poorly informed patients that might explain the distressingly low number of $10-15 \%$ of CTEPH cases that ultimately undergo PEA surgery according to a survey conducted in the EU and the USA (22). Probably the highest number of PEA's performed/year reported was $2.7 /$ million inhabitants by Papworth for 2017 (61). However, with an estimated incidence of CTEPH of $17 /$ million inhabitants a year and by considering at least $60 \%$ of these patients to be operable, in an ideal world you might assume that at least ten PEA surgeries/million inhabitants a year should be performed.

The advent of new interventional and medical therapies will complicate things further, and the place of each of them and their combination (multimodality approach) within the treatment strategy of CTEPH should be defined. However, the finding that too few patients undergo PEA surgery will undoubtedly persist. So the challenges for the upcoming decade(s) for the CTEPH community are not sparse.

\section{Conclusions}

Healthcare providers outside CTEPH centers having 
misinformation or insufficient education and a general lack of treatment awareness contribute to a massive underdiagnosis of CTEPH, diagnostic delay, and refusal of surgery by patients. Together with the subjective operability assessment, this leads to too few patients undergoing PEA, although this surgery results in an improved survival and exercise capacity. Acute PE survivors should be screened for CTEPH. Patients screened positive, and those with CTEPH symptoms (with or without history of PE) should undergo TTE to determine the probability of PH. High $\mathrm{PH}$ probability patients should undergo a V/Q scan. A negative scan rules out CTEPH. Patients with a positive V/Q scan but also patients with findings suggestive for CTEPH on CTPA performed to diagnose acute PE should be referred to a CTEPH center. Further diagnostic work-up currently consists of catheter based pulmonary angiography, CTPA and right heart catheterization. However, new imaging technologies might replace them in the near future, with one single imaging tool to screen, diagnose and assess operability as the ultimate goal. Operability assessment should be performed by a multidisciplinary CTEPH team. PEA surgery should be organized in a single center per country or for each forty to fifty million inhabitants in order to offer the highest level of expertise. In cases of non-operability assessment by a smaller center, a second opinion from a higher volume CTEPH center is recommended. Informing patients about PEA should preferably be done by the treating surgeon. Based on the estimated incidence of CTEPH and with a better education of patients and healthcare providers, despite the advent of new interventional and medical therapies for CTEPH, the number of PEA surgeries performed should still have the potential to grow significantly.

\section{Acknowledgments}

Funding: None.

\section{Footnote}

Conflicts of Interest: The authors have no conflicts of interest to declare.

Open Access Statement: This is an Open Access article distributed in accordance with the Creative Commons Attribution-NonCommercial-NoDerivs 4.0 International License (CC BY-NC-ND 4.0), which permits the noncommercial replication and distribution of the article with the strict proviso that no changes or edits are made and the original work is properly cited (including links to both the formal publication through the relevant DOI and the license). See: https://creativecommons.org/licenses/by-nc-nd/4.0/.

\section{References}

1. Ende-Verhaar YM, Cannegieter SC, Vonk Noordegraaf A, et al. Incidence of chronic thromboembolic pulmonary hypertension after acute pulmonary embolism: a contemporary view of the published literature. Eur Respir J 2017;49:1601792.

2. Mahmud E, Madani MM, Kim NH, et al. Chronic Thromboembolic Pulmonary Hypertension: Evolving Therapeutic Approaches for Operable and Inoperable Disease. J Am Coll Cardiol 2018;71:2468-86.

3. Delcroix M, Torbicki A, Gopalan D, et al. ERS statement on chronic thromboembolic pulmonary hypertension. Eur Respir J 2021;57:2002828.

4. van der Plas MN, Reesink HJ, Roos CM, et al. Pulmonary endarterectomy improves dyspnea by the relief of dead space ventilation. Ann Thorac Surg 2010;89:347-52.

5. Papamatheakis DG, Poch DS, Fernandes TM, et al. Chronic Thromboembolic Pulmonary Hypertension: JACC Focus Seminar. J Am Coll Cardiol 2020;76:2155-69.

6. Riedel M, Stanek V, Widimsky J, et al. Longterm followup of patients with pulmonary thromboembolism. Late prognosis and evolution of hemodynamic and respiratory data. Chest 1982;81:151-8.

7. Delcroix M, Lang I, Pepke-Zaba J, et al. Long-Term Outcome of Patients With Chronic Thromboembolic Pulmonary Hypertension: Results From an International Prospective Registry. Circulation 2016;133:859-71.

8. Jenkins D, Madani M, Fadel E, et al. Pulmonary endarterectomy in the management of chronic thromboembolic pulmonary hypertension. Eur Respir Rev 2017;26:160111.

9. Taboada D, Pepke-Zaba J, Jenkins DP, et al. Outcome of pulmonary endarterectomy in symptomatic chronic thromboembolic disease. Eur Respir J 2014;44:1635-45.

10. van Kan C, van der Plas MN, Reesink HJ, et al. Hemodynamic and ventilatory responses during exercise in chronic thromboembolic disease. J Thorac Cardiovasc Surg 2016;152:763-71.

11. Galiè N, Humbert M, Vachiery JL, et al. 2015 ESC/ERS Guidelines for the diagnosis and treatment of pulmonary hypertension: The Joint Task Force for the Diagnosis and Treatment of Pulmonary Hypertension of the 
European Society of Cardiology (ESC) and the European Respiratory Society (ERS): Endorsed by: Association for European Paediatric and Congenital Cardiology (AEPC), International Society for Heart and Lung Transplantation (ISHLT). Eur Heart J 2016;37:67-119.

12. Konstantinides SV, Meyer G, Becattini C, et al. 2019 ESC Guidelines for the diagnosis and management of acute pulmonary embolism developed in collaboration with the European Respiratory Society (ERS). Eur Heart J 2020;41:543-603.

13. Houk VN, Hufnagel CA, Mcclenathan JE, et al. Chronic thrombotic obstruction of major pulmonary arteries. report of a case successfully treated by thrombendarterectomy, and a review of the literature. Am J Med 1963;35:269-82.

14. Panasiuk A, Dziecioł J, Nowak HF, et al. Pulmonary thromboembolism--random analysis of autopsy material. Pneumonol Alergol Pol 1993;61:171-6.

15. Goldhaber SZ, Visani L, De Rosa M. Acute pulmonary embolism: clinical outcomes in the International Cooperative Pulmonary Embolism Registry (ICOPER) Lancet 1999;353:1386-9.

16. Guérin L, Couturaud F, Parent F, et al. Prevalence of chronic thromboembolic pulmonary hypertension after acute pulmonary embolism. Prevalence of CTEPH after pulmonary embolism. Thromb Haemost 2014;112:598-605.

17. Pepke-Zaba J, Delcroix M, Lang I, et al. Chronic thromboembolic pulmonary hypertension (CTEPH): results from an international prospective registry. Circulation 2011;124:1973-81.

18. Kramm T, Wilkens H, Fuge J, et al. Incidence and characteristics of chronic thromboembolic pulmonary hypertension in Germany. Clin Res Cardiol 2018;107:548-53.

19. Delcroix M, Kerr K, Fedullo P. Chronic Thromboembolic Pulmonary Hypertension. Epidemiology and Risk Factors. Ann Am Thorac Soc 2016;13 Suppl 3:S201-6.

20. Klok FA, Barco S, Konstantinides SV, et al. Determinants of diagnostic delay in chronic thromboembolic pulmonary hypertension: results from the European CTEPH Registry. Eur Respir J 2018;52:1801687.

21. Fedullo PF, Kerr KM, Auger WR. Pulmonary hypertension due to pulmonary embolism and thromboembolic obstruction of proximal and distal pulmonary arteries. In: Yuan JXJ, Garcia JGN, Hales CA, et al. editors. Textbook of pulmonary vascular disease. New York: Springer; 2011:1239-51.

22. Gall H, Preston IR, Hinzmann B, et al. An international physician survey of chronic thromboembolic pulmonary hypertension management. Pulm Circ 2016;6:472-82.

23. Klok FA, van Kralingen KW, van Dijk AP, et al. Prospective cardiopulmonary screening program to detect chronic thromboembolic pulmonary hypertension in patients after acute pulmonary embolism. Haematologica 2010;95:970-5.

24. Klok FA, van Kralingen KW, van Dijk AP, et al. Prevalence and potential determinants of exertional dyspnea after acute pulmonary embolism. Respir Med 2010;104:1744-9.

25. Boon GJAM, Ende-Verhaar YM, Bavalia R, et al. Noninvasive early exclusion of chronic thromboembolic pulmonary hypertension after acute pulmonary embolism: the InShape II study. Thorax 2021;76:1002-9.

26. Ende-Verhaar YM, Huisman MV, Klok FA. To screen or not to screen for chronic thromboembolic pulmonary hypertension after acute pulmonary embolism. Thromb Res 2017;151:1-7.

27. Klok FA, Couturaud F, Delcroix M, et al. Diagnosis of chronic thromboembolic pulmonary hypertension after acute pulmonary embolism. Eur Respir J 2020;5 5:2000189.

28. Held M, Grün M, Holl R, et al. Cardiopulmonary exercise testing to detect chronic thromboembolic pulmonary hypertension in patients with normal echocardiography. Respiration 2014;87:379-87.

29. Claessen G, La Gerche A, Voigt JU, et al. Accuracy of Echocardiography to Evaluate Pulmonary Vascular and RV Function During Exercise. JACC Cardiovasc Imaging 2016;9:532-43.

30. Kiely DG, Levin D, Hassoun P, et al. EXPRESS: Statement on imaging and pulmonary hypertension from the Pulmonary Vascular Research Institute (PVRI). Pulm Circ 2019. [Epub ahead of print].

31. Tunariu N, Gibbs SJ, Win Z, et al. Ventilation-perfusion scintigraphy is more sensitive than multidetector CTPA in detecting chronic thromboembolic pulmonary disease as a treatable cause of pulmonary hypertension. J Nucl Med 2007;48:680-4.

32. Auger WR, Fedullo PF, Moser KM, et al. Chronic majorvessel thromboembolic pulmonary artery obstruction: appearance at angiography. Radiology 1992;182:393-8.

33. Rogberg AN, Gopalan D, Westerlund E, et al. Do radiologists detect chronic thromboembolic disease on computed tomography? Acta Radiol 2019;60:1576-83.

34. Sugiura T, Tanabe N, Matsuura Y, et al. Role of 320-slice CT imaging in the diagnostic workup of patients with chronic thromboembolic pulmonary hypertension. Chest 
2013;143:1070-7.

35. Roach PJ, Schembri GP, Bailey DL. V/Q scanning using SPECT and SPECT/CT. J Nucl Med 2013;54:1588-96.

36. Kligerman S, Hsiao A. Optimizing the diagnosis and assessment of chronic thromboembolic pulmonary hypertension with advancing imaging modalities. Pulm Circ 2021;11:20458940211007375.

37. Soler X, Hoh CK, Test VJ, et al. Single photon emission computed tomography in chronic thromboembolic pulmonary hypertension. Respirology 2011;16:131-7.

38. Wirth G, Brüggemann K, Bostel T, et al. Chronic thromboembolic pulmonary hypertension (CTEPH) potential role of multidetector-row CT (MD-CT) and MR imaging in the diagnosis and differential diagnosis of the disease. Rofo 2014;186:751-61.

39. Hedgire SS, Baliyan V, Ghoshhajra BB, et al. Recent advances in cardiac computed tomography dose reduction strategies: a review of scientific evidence and technical developments. J Med Imaging (Bellingham) 2017;4:031211.

40. Kröger JR, Gerhardt F, Dumitrescu D, et al. Diagnosis of pulmonary hypertension using spectral-detector CT. Int J Cardiol 2019;285:80-5.

41. Hoey ET, Mirsadraee S, Pepke-Zaba J, et al. Dual-energy CT angiography for assessment of regional pulmonary perfusion in patients with chronic thromboembolic pulmonary hypertension: initial experience. AJR Am J Roentgenol 2011;196:524-32.

42. Dong C, Zhou M, Liu D, et al. Diagnostic accuracy of computed tomography for chronic thromboembolic pulmonary hypertension: a systematic review and metaanalysis. PLoS One 2015;10:e0126985.

43. Kreitner KF, Kunz RP, Ley S, et al. Chronic thromboembolic pulmonary hypertension - assessment by magnetic resonance imaging. Eur Radiol 2007;17:11-21.

44. Pöhler GH, Klimes F, Voskrebenzev A, et al. Chronic Thromboembolic Pulmonary Hypertension Perioperative Monitoring Using Phase-Resolved Functional Lung (PREFUL)-MRI. J Magn Reson Imaging 2020;52:610-9.

45. Wilkens H, Konstantinides S, Lang IM, et al. Chronic thromboembolic pulmonary hypertension (CTEPH): Updated Recommendations from the Cologne Consensus Conference 2018. Int J Cardiol 2018;272S:69-78.

46. Madani M, Mayer E, Fadel E, et al. Pulmonary Endarterectomy. Patient Selection, Technical Challenges, and Outcomes. Ann Am Thorac Soc 2016;13 Suppl 3:S240-7.
47. Jenkins DP, Biederman A, D'Armini AM, et al. Operability assessment in CTEPH: Lessons from the CHEST-1 study. J Thorac Cardiovasc Surg 2016;152:669-674.e3.

48. Mayer E, Jenkins D, Lindner J, et al. Surgical management and outcome of patients with chronic thromboembolic pulmonary hypertension: results from an international prospective registry. J Thorac Cardiovasc Surg 2011;141:702-10.

49. Madani MM, Auger WR, Pretorius V, et al. Pulmonary endarterectomy: recent changes in a single institution's experience of more than 2,700 patients. Ann Thorac Surg 2012;94:97-103; discussion 103.

50. Korsholm K, Andersen A, Mellemkjær S, et al. Results from more than 20 years of surgical pulmonary endarterectomy for chronic thromboembolic pulmonary hypertension in Denmark. Eur J Cardiothorac Surg 2017;52:704-9.

51. Zhang L, Bai Y, Yan P, et al. Balloon pulmonary angioplasty vs. pulmonary endarterectomy in patients with chronic thromboembolic pulmonary hypertension: a systematic review and meta-analysis. Heart Fail Rev 2021;26:897-917.

52. Olgun Yıldızeli Ş, Kepez A, Taş S, et al. Pulmonary endarterectomy for patients with chronic thromboembolic disease. Anatol J Cardiol 2018;19:273-8.

53. Claeys M, Claessen G, La Gerche A, et al. Impaired Cardiac Reserve and Abnormal Vascular Load Limit Exercise Capacity in Chronic Thromboembolic Disease. JACC Cardiovasc Imaging 2019;12:1444-56.

54. Madani MM, Wittine LM, Auger WR, et al. Chronic thromboembolic pulmonary hypertension in pediatric patients. J Thorac Cardiovasc Surg 2011;141:624-30.

55. Verbelen T, Cools B, Fejzic Z, et al. Pulmonary endarterectomy in a 12 -year-old boy with multiple comorbidities. Pulm Circ 2019;9:2045894019886249.

56. Grazioli V, Ghio S, Pin M, et al. Pulmonary endarterectomy in the octogenarian population: safety and outcomes. J Cardiovasc Med (Hagerstown) 2021;22:567-71.

57. Pandey AK, Lee NS, Marsal J, et al. Evaluation of Routine Coronary Angiography Before Pulmonary Thromboendarterectomy. Ann Thorac Surg 2021;111:1703-9.

58. Kim NH, Delcroix M, Jenkins DP, et al. Chronic thromboembolic pulmonary hypertension. J Am Coll Cardiol 2013;62:D92-9.

59. Cannon JE, Su L, Kiely DG, et al. Dynamic Risk 
Stratification of Patient Long-Term Outcome After Pulmonary Endarterectomy: Results From the United Kingdom National Cohort. Circulation 2016;133:1761-71.

60. Quadery SR, Swift AJ, Billings CG, et al. The impact of patient choice on survival in chronic thromboembolic

Cite this article as: Verbelen T, Godinas L, Maleux G, Coolen J, Claessen G, Belge C, Meyns B, Delcroix M. Chronic thromboembolic pulmonary hypertension: diagnosis, operability assessment and patient selection for pulmonary endarterectomy. Ann Cardiothorac Surg 2022;11(2):82-97. doi: 10.21037/acs2021-pte-12 pulmonary hypertension. Eur Respir J 2018;52:1800589.

61. Condliffe R, Quadery SR, Jenkins DP, et al. Decisionmaking in pulmonary endarterectomy surgery. Eur Respir J 2019;53:1801973. 